\title{
TRAIN LOCALIZATION USING AN ADAPTIVE MULTISENSOR DATA FUSION TECHNIQUE
}

\author{
Bidhan MALAKAR*, Binoy Krishna ROY \\ Dept of Electrical Engineering, National Institute of Technology, Silchar, India \\ Received 12 April 2016; revised 5 July 2016; accepted 17 August 2016
}

\begin{abstract}
This work deals with the development of an adaptive multisensor data fusion technique for the accurate estimation of the trains position and velocity. The proposed technique will work with the Train Collision Avoidance System (TCAS) used in Indian railways during Global Positioning System (GPS) outages. The determination of accurate position of trains is a challenging task for the TCAS during GPS outages. The accuracy of the proposed Volterra Recursive Least Square (VRLS) based adaptive multisensor data fusion technique is evaluated by generating two kinematic profiles for a passenger train running between Silchar-Lumding broad gauge route in Indian railways. The effect of accelerometer bias is also considered during the analysis. It is observed that the developed technique can provide a better estimate of the position and velocity for the TCAS especially during GPS outages and without using any additional railway infrastructure. The simulation results indicate that the proposed technique is superior to the earlier works in terms of achieving better positional accuracy in presence of accelerometer bias.
\end{abstract}

Keywords: train positioning, multisensor data fusion, signal processing, train collision avoidance system, global positioning system, odometer, accelerometer.

\section{Introduction}

Multisensor data fusion technique refers to the process of combining observations from different sensors to improve the quality of the information of a process of interest so that in some sense the overall effect is greater than the sum of effects if considered individually (Mitchell 2007). The use of multisensor data fusion techniques also gained much popularity and found to be advantageous when used in intelligent transportation system (Walts 1988; El Faouzi et al. 2011; Bachmann et al. 2012; Khaleghi et al. 2013) especially in control and guidance of autonomous vehicles (Gregor et al. 2002; Chen, Han 2005).

In the recent years, the research related to the field of train positioning system is gaining more attention (Santos et al. 2005; Chang, Tsang 2008; Arunachalam et al. 2013; Hartwig et al. 2006). The positioning system can help the railway engineers in achieving higher safety. The navigation system becomes robust when the multisensor data fusion techniques are included in the system. The determination of the position of the train can be used to avoid major collisions viz. the Gaisal train accident in India.

The use of GPS for land vehicle navigation purposes have also increased due to its miniature size and low cost
(Qin et al. 2012). However, the use of GPS got increased but, still there are many inherent shortcomings of GPS while using in the land vehicle positioning system (Zhao 2011). The satellite-based GPS requires a line-of-sight between the satellites and receiver antenna. A good quality line-of-sight is always not guaranteed in case when a land vehicle is travelling through dense forest areas, mountainous regions that hinders the satellite signals from reaching the antenna. The signal acquisition and tracking becomes difficult during GPS outages and which in turn affects the GPS navigation accuracy (Kaplan 1996). Thus, there is a requirement of an efficient technique that can be included in the train navigation system that will help in precisely localising the trains in case of unavailability of GPS information.

The TCAS is an autonomous system developed by Indian railways and is able to provide the location information of trains using GPS (Mansukhani 2014). One of the major issue of the TCAS is the requirement of good quality GPS signal, which helps in locating the trains. A case study of the trains running in the Silchar-Lumding broad gauge route a hilly region in Indian railways is

${ }^{*}$ Corresponding author. E-mail: bidhan.nits@gmail.com 
chosen for the analysis. The problem associated with the phenomenon of GPS jamming due to the interference in signal coming from the satellite is one of the common problem occurring in this hilly route (Kim et al. 2011; Prakash et al. 2008). Moreover, there are also certain limitations with the techniques that are being used at present for the train localization. The use of Kalman filter in train navigation system is reported by several researchers (Mirabadi et al. 2003; Ernest et al. 2004; Acharya et al. 2011). The working of Kalman filter mainly depends on a set of measurements and also it requires a proper initial knowledge of the dynamic model of the navigation system to provide an optimal estimation of the states. An optimal estimation is not guaranteed in presence of nonlinearities and also in case the input data does not fit well with the model (Kalman 1960). This problem can be solved by using an Extended Kalman Filter (EKF) (Cooper et al. 1994), but again the EKF works on the principle of linearization of the state transition matrix and the observation matrix, which can degrade the performance and divergence of the filter for highly non-linear systems (Ribeiro 2004). Hence, the investigation of a new adaptive multisensor data fusion technique for the TCAS based train navigation system becomes the motivation of this research.

The objectives of the present work are as follows:

- to develop a localization technique for the TCAS used in Indian railways that can accurately localize the trains during GPS outages;

- to analyse the proposed VRLS based adaptive multisensor data fusion technique by considering a case study of a train running in the Silchar-Lumding broad gauge route in Indian railways;

- to study the comparative performances of the proposed technique with three other available techniques considering two different kinematic profiles and including the effect of various accelerometer bias and noise adopted from (Noureldin et al. 2013; Groves 2013).

\section{An overview of TCAS in Indian railways}

The TCAS is developed under Automatic Train Protection System (ATP) that works as an autonomous system and is capable of providing the longitudinal position information of the running trains (Mansukhani 2014). The design specifications as shown in Figure 1 are based on European Train Control System (ETCS) Level-I as per Research Design and Standard Organisation (RDSO) (Mangal 2013).

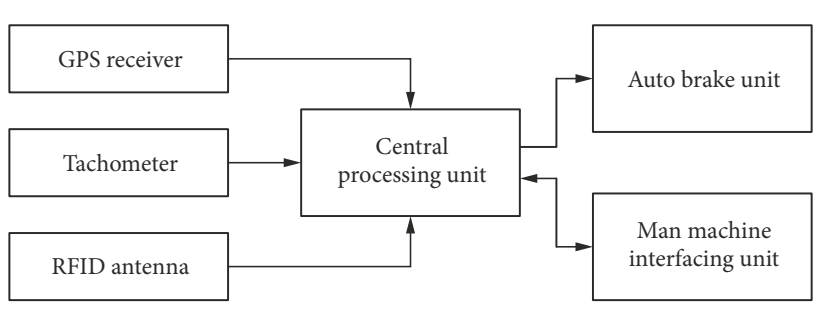

Figure 1. Architecture of the TCAS installed in locomotive
Quality assessment and short trials with the TCAS have been conducted in the joint supervision of RDSO and M/s HBL, Hyderabad in 2012 in Southern region of Indian railways (Mangal 2013).

Some of the shortcomings with the current version of TCAS are given below:

- the use of Radio-Frequency IDentification (RFID) tags in determining the position of the train in this kind of harsh environments may not be a suitable choice and there are certain limitations as discussed in (Zhang et al. 2010); a brief survey of RFID application in railway industry is presented in (Malakar, Roy 2014);

- the error related to the GPS or Global Navigation Satellite System (GNSS) receiver may increase whenever the train passes through dense forest, tunnels or GPS dark regions Bajaj et al. (2002);

- the degraded adhesion between the wheel and rail contacts, which commonly occurs in this kind of hilly routes may affect the performance of tachometer (Wang et al. 2014; Government of India 2012).

\section{Dynamic modelling}

In this work, the Wiener-process acceleration model is used to derive the discrete state equations (Bar-Shalom, Li 1993). The Wiener process acceleration model assumes the acceleration to be a Wiener process (Bar-Shalom, Li 1993). The corresponding discrete-time equivalent state equation with a sampling time interval of $\Delta \mathrm{t}$ is given by:

$$
x_{k+1}=F_{k} \cdot x_{k}+P \cdot a_{k}+w_{k},
$$

where:

$$
F_{k}=\left(\begin{array}{ccc}
1 & \Delta t & \frac{\Delta t^{2}}{2} \\
0 & 1 & \Delta t \\
0 & 0 & 1
\end{array}\right)
$$

and is the transition matrix:

$$
\begin{gathered}
P=\left(\begin{array}{c}
\frac{\Delta t^{2}}{2} \\
\Delta t \\
0
\end{array}\right) ; \\
x_{k}=\left[\begin{array}{l}
d_{k} \\
v_{k} \\
b_{k}
\end{array}\right] .
\end{gathered}
$$

In Equation (1) above, the three state variables are $d_{k}$, $v_{k}$ and $b_{k}$, which represent the position, velocity and bias of the accelerometer respectively. In Equation (1), the acceleration $a_{k}$ measured by the accelerometer at the $k$-th sample is assumed to be a known input. The quantity $a_{k}-$ $b_{k}$ gives the measure of true acceleration. It is obvious that this kind of choices works effectively as described in researches by Ernest et al. (2004) and Acharya et al. (2011). The accelerometer used in this work not only acquire the 
true kinematic acceleration profile but at the same time also includes the components of:

- acceleration due to gravity;

- disturbances raised from the vibrations and angular accelerations; also, the effects of climbs and descends in a grade is continuously compensated and is included in the analysis.

The covariance matrix $Q_{k}$ with the discrete-time process noise $w_{k}$ is given by:

$$
Q_{k}=\operatorname{cov}\left(w_{k}\right)=\sigma_{w}^{2} \cdot T_{3} \text {, }
$$

where: $\sigma_{w}^{2}$ represents the autocorrelation of the scalar white noise $w_{k}$;

$$
T_{3}=\left(\begin{array}{ccc}
\frac{\Delta t^{5}}{20} & \frac{\Delta t^{4}}{8} & \frac{\Delta t^{3}}{6} \\
\frac{\Delta t^{4}}{8} & \frac{\Delta t^{3}}{3} & \frac{\Delta t^{2}}{2} \\
\frac{\Delta t^{3}}{6} & \frac{\Delta t^{2}}{2} & \Delta t
\end{array}\right) .
$$

The output equation by considering the Gaussian measurement noise $e_{k}$ is given by:

$$
z_{k}=\left(\begin{array}{lll}
0 & 1 & 0 \\
0 & 0 & 1
\end{array}\right) \cdot\left[\begin{array}{l}
d_{k} \\
v_{k} \\
b_{k}
\end{array}\right]+e_{k} .
$$

\section{Proposed technique for localization of trains}

In this work, a localization system is developed for the TCAS to determine the position of trains during the unavailability of GPS information without relying on any trackside aids.

The proposed data fusion technique for train localization system consists of three on-board sensors namely longitudinal accelerometer, odometer and GPS receiver unit that are installed in the locomotive. The data fusion scheme for the navigation system installed in the locomotive serves as a means of providing the longitudinal position of the train along the track. The working of the proposed Localization system is shown in the Figure 2.

The slip and slide errors due to poor wheel-rail adhesion are very common in this hilly regions and are included in the analysis while estimating the position and velocity during GPS outages (Mirabadi et al. 2002). The slip and slide errors modelled by $e_{k}$ in Equation (3) can also affect the performance of the odometer.

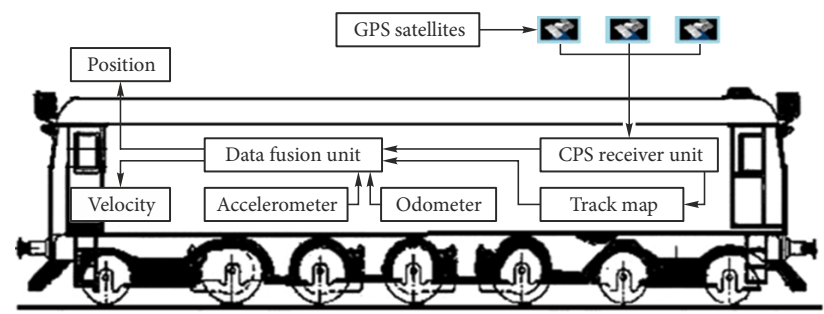

Figure 2. Working of the proposed localization system
Following are the two cases considered when poor adhesion between the wheel and rail occurs and which are as follows:

- when there is no slip and slide error modelled by $e_{k}$ occurs in the odometer, then the adaptive filter is able to estimate the optimal accelerometer bias and by integrating this bias value one can get the velocity and again integrating gives the position of the train;

- when the slip and slide error modelled by $e_{k}$ is large the true velocity measurement from the odometer can be ignored by choosing a large measurement noise covariance in the adaptive filter and the velocity and position can be obtained from using only the acceleration measurement.

These two above considered cases will work only if one could determine when $e_{k}$ is having a larger value. This kind of assumptions are already found to be useful for the fusion strategies as given in the researches by Ernest $e t$ al. (2004) and Acharya et al. (2011).

The fourth source of information is provided by the digital map, which includes the geographical and geometrical information of the railway network. In case of the unavailability of GPS information the position is estimated by the on-board system installed in the locomotive.

Thus, an analysis will be carried out for the TCAS using the above sensors configuration to evaluate the performance of the proposed technique in the following subsections.

\subsection{Adaptive datafusion scheme for localization of trains}

An adaptive filtering technique is mainly concerned with designing of time-varying adaptive filters capable of tuning themselves to optimally process the non-stationary signals in a dynamic environment (Diniz 2013). Adaptive filters are proved to be one of the promising technique when applied in case of non-stationary systems and noisy environments. Adaptive filters are also successfully implemented in several applications that include: (1) system identification, (2) inverse system modelling, (3) signal prediction and interference cancelation. A combination of the data fusion with the proposed adaptive algorithm for the Indian railway TCAS is shown in Figure 3. The filter coefficients are obtained by the adaptive filtering algorithm, which in turn accurately estimates the mean square of the position and velocity error.

The error $e(n)$ is calculated as $d(n)-y(n)$. An objective function is developed using this error information that is required by the adaptation algorithm in order to determine the appropriate updating of the filter coefficients. After going through various iterative processes, if there is a minimization of the objective function then it can be concluded that the adaptive-filter output is matching with the desired position and velocity. The adaptive algorithm is mainly responsible for the adjustment of the adaptivefilter coefficients and which generally help in the reduction of the prescribed criterion. 


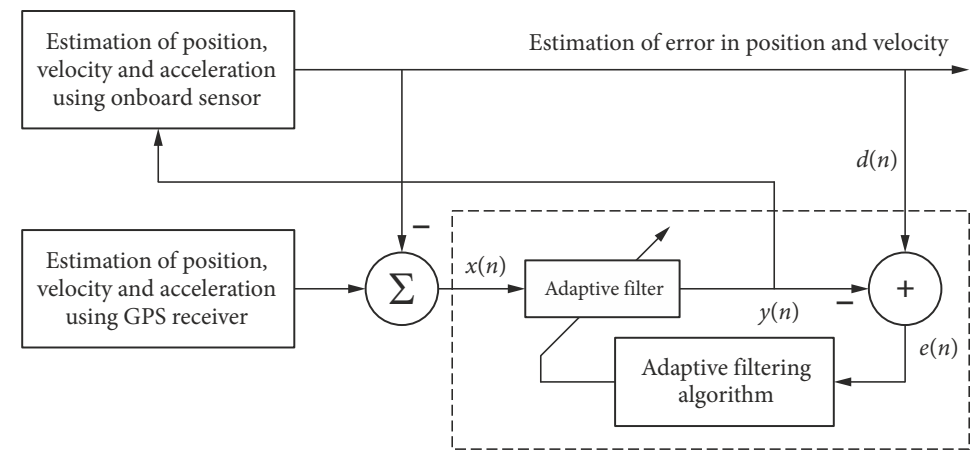

Figure 3. Multisensor datafusion scheme for train localization: $n$ - iteration number; $x(n)$ - the adaptive-filter input; $y(n)$ - adaptive-filter output; $d(n)$ - the desired position and velocity; $e(n)$ - error

\subsection{VRLS algorithm}

The Volterra series model is widely accepted and is found to be one of the most promising model for nonlinear systems (Diniz 2013).

According to the Volterra series expansion, the relationship between the input and output of a nonlinear system consisting of non iterative series is given by:

$$
\begin{aligned}
d^{\prime}(k)= & \sum_{l_{1}=0}^{\infty} w_{0}\left(l_{1}\right) \cdot x\left(k-l_{1}\right)+ \\
& \sum_{l_{1}=0}^{\infty} \sum_{l_{2}=0}^{\infty}\left(l_{1}, l_{2}\right) \cdot x\left(k-l_{1}\right) \cdot x\left(k-l_{2}\right)+ \\
& \sum_{l_{1}=0}^{\infty} \sum_{l_{2}=0}^{\infty} \sum_{l_{3}=0}^{\infty} w_{03}\left(l_{1}, l_{2}, l_{3}\right) \cdot x\left(k-l_{1}\right) \times \\
& x\left(k-l_{2}\right) \cdot x\left(k-l_{3}\right)+ \\
& \sum_{l_{1}=0}^{\infty} \sum_{l_{2}=0}^{\infty} \ldots \sum_{l_{i}=0}^{\infty} w_{0 i}\left(l_{1}, l_{2}, \ldots, l_{i}\right) \cdot x\left(k-l_{1}\right) \times \\
& x\left(k-l_{2}\right)+\ldots+x\left(k-l_{i}\right)+\ldots,
\end{aligned}
$$

where: $w_{0 i}\left(l_{1}, l_{2}, \ldots, l_{i}\right)$, for $i=0,1, \ldots, \infty$, are the coefficients of the nonlinear filter model based on the Volterra series; $d^{\prime}(k)$ is used for system identification purpose and represents the unknown system output in absence of measurement noise.

The necessary computational steps required by the VRLS algorithm for updating the weights of the unknown parameters is given below:

- Step 1. Initialization (set parameters for vector initialization):

$$
S_{D}(-1)=\delta I \text {, }
$$

where: $\delta$ - inverse of an estimate of the position and velocity; $S_{D}$ - inverse of the deterministic correlation matrix of the input vector; $I$ - identity matrix;

- Step 2. Compute the true kinematic acceleration profile by considering various slip factors;

- Step 3. Compute the measured acceleration: measured acceleration $=$ true acceleration + accelerometer bias + accelerometer noise;
- Step 4. Compute the velocity from odometer: velocity $=($ true velocity + slip $) \times$ odometer scale factor $(S F)$;

- Step 5. Assign the filter parameters $Q, R$ and $v$;

- Step 6. $x(-1)=w(-1)=\left[\begin{array}{llll}0 & 0 & \ldots & 0\end{array}\right]^{T}$, do for $n \geq 0$;

- Step 7. Calculate $e(k)$ using the relation: $e(n)=d(n)-x^{T}(n) \cdot w(n-1)$, where: $e(n)$ is the error;

- Step 8. Calculate: $\psi(n)=S_{D}(n-1) \cdot x(n)$, where: $x(n)$ is input vector;

- Step 9. Calculate inverse of the deterministic correlation matrix of the input vector:

$$
S_{D}(n)=\frac{1}{\lambda} \cdot \frac{S_{D}(n-1)-\psi(n) \cdot \psi^{T}(n)}{\lambda+\psi^{T}(n) \cdot x(n)},
$$

where: $\lambda$ is an exponential weighting factor that should be chosen in the range of $0 \leq \lambda \leq 1$;

- Step 10. Update $w$ matrix:

$$
w(n)=w(n-1)+e(n) \cdot S_{D}(n) \cdot x(n),
$$

where: $w(n)$ are coefficients of the non-linear filter model based on the second order of the Volterra series expansion;

- Step 11. If necessary, compute $y(n)$ matrix:

$$
y(n)=w^{T}(n) \cdot x(n),
$$

where: $y(n)$ is adaptive filter output;

$$
\varepsilon(n)=d(n)-y(n) \text {, }
$$

where: $\varepsilon(n)$ is mean square error.

The selection of the suitable coefficients of the adaptive filter is done by the Recursive Least Square (RLS) algorithm so that the output $y(n)$, will try to match the desired position and velocity in the least-squares sense. The process of minimization is similar to the Least Mean Square (LMS) case and can be adjusted easily for the nonlinear adaptive filtering case through reinterpretation of the entries of the system input vector and the coefficient vector (Diniz 2013).

As discussed earlier, the TCAS is a satellite based navigation system, which employs RFID tags and GPS or GNSS that basically assist to locate the trains on a track. Both of these systems are found to be unsuitable for the 
trains running in the northeast region of Indian railways due to various environmental factors and may result in poor positional accuracy (Government of India 2015). On the other hand, the accuracy of tachometer for measuring the speed especially in this kind of hilly routes is also suffered by the error generated by the slipping and sliding due to wheel-rail contacts (Mirabadi et al. 2002; Geistler, Bohringer 2004). It is observed from the present analysis that VRLS based adaptive multisensor data fusion technique can effectively estimate the position and velocity in presence of accelerometer bias and noise.

\section{Dynamic simulation of the localization system}

\subsection{Kinematic profile}

The kinematic profile is obtained for a passenger train running between Silchar-Lumding route in the NorthEastern region of Indian railway. The accuracy of the proposed technique is analysed for two different kinematic profiles that are generated for two different journeys. The assumed kinematic profiles that are obtained for the passenger train while passing through two different stations is given in Table 1 .

The acceleration profiles are generated for the two journeys named A and B for different accelerometer bias and noise and are given below in Table 2 .

The kinematic profile both true and estimated for all the cases including the bias and accelerometer noise are shown in Figure 4, and the technique discussed in research by Acharya et al. (2011), is mainly used to synthetically generate the profiles. The present journeys (Journey-A and Journey-B) also include acceleration, cruising, coasting, mild braking, deceleration and stop at different velocities.

The assumed kinematic profile can be further integrated to compute the distance travelled. The acceleration kicks as shown in the Figure 4 in the negative direction shows the phenomenon of the wheel slippage due to the poor wheel-rail adhesion, which is also considered during the analysis (Wang et al. 2014).

Table 1. Journey details of the passenger train

\begin{tabular}{|l|c|c|}
\hline Journey name & Distance $\sim[\mathrm{km}]$ & Travel time $\sim[\mathrm{h}: \mathrm{min}]$ \\
\hline Journey-A & 26 & $1: 20$ \\
\hline Journey-B & 46 & $1: 30$ \\
\hline
\end{tabular}

Table 2. Accelerometer bias and noise considered during the analysis for Journey-A and Journey-B

\begin{tabular}{|c|c|c|c|}
\hline $\begin{array}{c}\text { Journey } \\
\text { name }\end{array}$ & $\begin{array}{c}\text { Type of } \\
\text { profile }\end{array}$ & $\begin{array}{c}\text { Accelerometer } \\
\text { bias } A_{b}\left[\mathrm{~m} / \mathrm{s}^{2}\right]\end{array}$ & $\begin{array}{c}\text { Accelerometer } \\
\text { noise } A_{n}\left[\mathrm{~m} / \mathrm{s}^{2}\right]\end{array}$ \\
\hline \multirow{3}{*}{ Journey-A } & Profile-1 & 0.20 & 0.05 \\
\cline { 2 - 4 } & Profile-2 & 0.26 & 0.02 \\
\cline { 2 - 4 } & Profile-3 & 0.30 & 0.05 \\
\hline \multirow{3}{*}{ Journey-B } & Profile-4 & 0.10 & 0.02 \\
\cline { 2 - 4 } & Profile-5 & 0.20 & 0.01 \\
\cline { 2 - 4 } & Profile-6 & 0.25 & 0.01 \\
\hline
\end{tabular}

a)
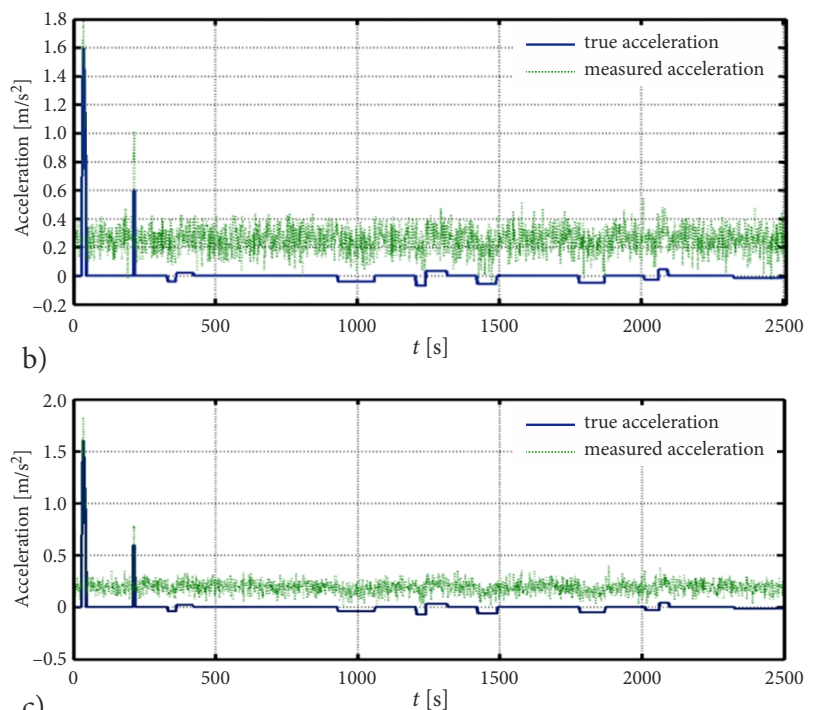

c)

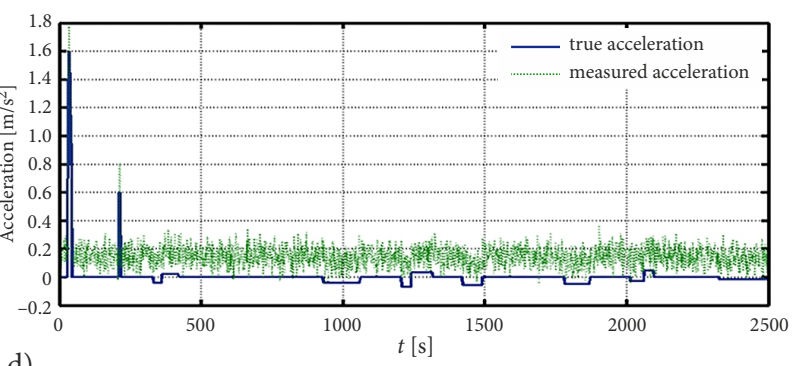

d)

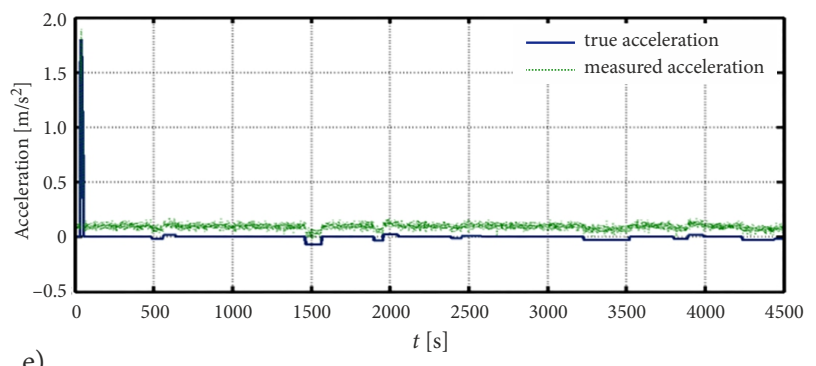

e)

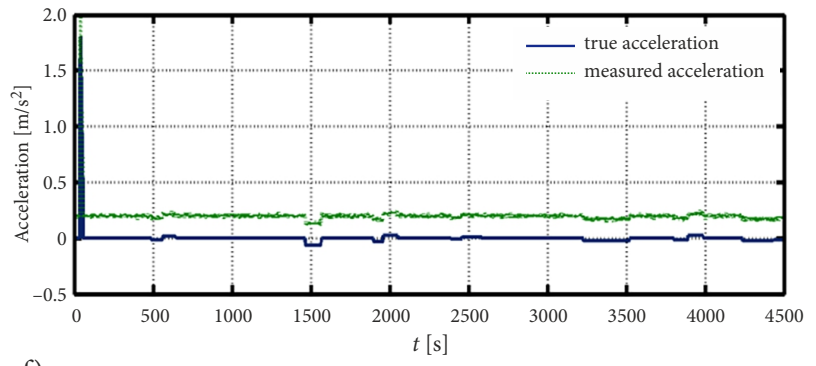

f)

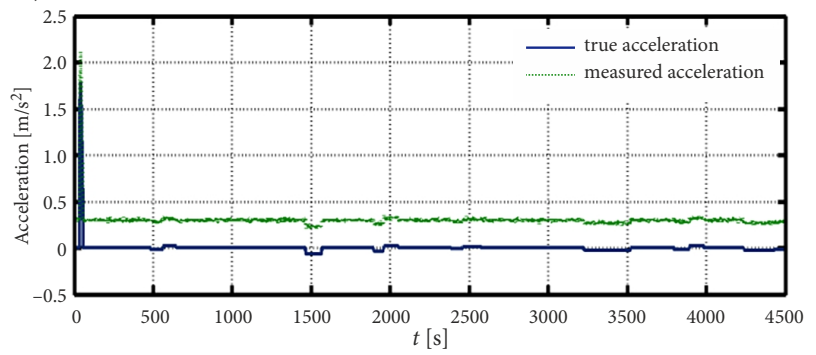

Figure 4. Kinematic profiles for journeys with profiles: a - Journey-A with Profile-1; b - Journey-A with Profile-23; c - Journey-A with Profile-3; d - Journey-B with Profile-4; e - Journey-B with Profile-5; f - Journey-B with Profile-6 
a)

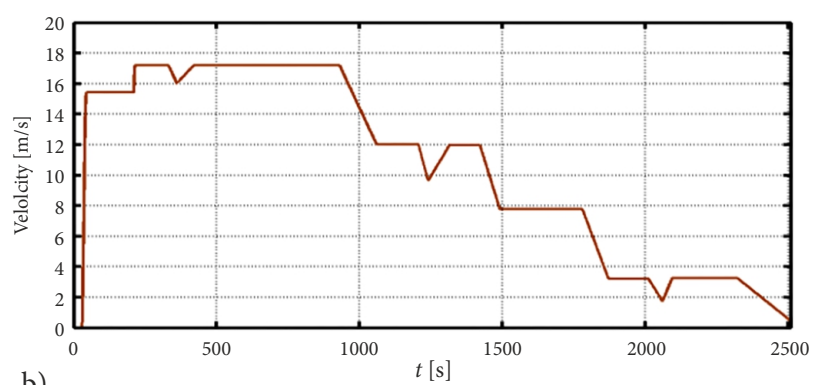

b)

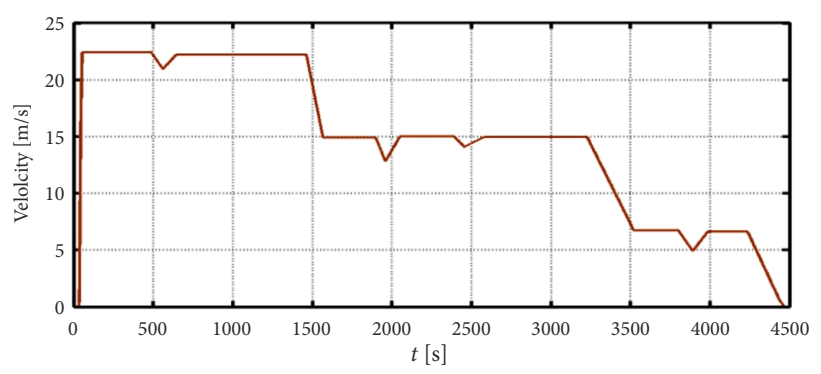

Figure 5. Velocity profiles for journeys: a - Journey-A; b - Journey-B

The true velocity obtained from the assumed kinematic profiles is shown in Figure 5. For the comparison of various results, the odometric profile given in research by Acharya et al. (2011) is used.

\subsection{Dynamic simulation for performance analysis}

\subsubsection{Estimation of velocity error}

The position and velocity are estimated using the VRLS based adaptive multisensor data fusion technique in presence of three different accelerometer bias and noise each for Journey-A and Journey-B as given in Table 2. The results of the comparative performances of the available techniques for the representative cases along with the proposed one with respect to the velocity estimation error are shown in Figure 6 and rest of the results are given in Table 3. The best values of filter parameters for VRLS technique are determined experimentally on a trial and error basis, i.e. $Q=0.5 \mathrm{~m}^{2} / \mathrm{s}^{4}, R=10 \mathrm{~m} / \mathrm{s}^{2}, v=0.01 \mathrm{~m} / \mathrm{s}^{2}$ and odometer scale factor $S F=0.98$. In Figure 6 , the time scale for some selected regions are enhanced for a better visibility. The comparative performance of the fusion-filtering techniques for velocity estimation error for the assumed kinematic profiles are presented in Table 3.

It can be observed from Table 3 that the absolute peak velocity error estimated by the VRLS based adaptive multisensor data fusion technique for the assumed kinematic profiles with various bias and accelerometer noise along with larger wheel slippage is much lesser when compared with the Observation Error Based Approach (OEBA), Bounded Offset Based Approach (BOBA) and PseudoMeasurement State Constraining (PMSC) techniques. The detailed results of the analysis are given in Table 3. It further reveals some interesting information regarding the performance of the proposed technique while estimat- a)

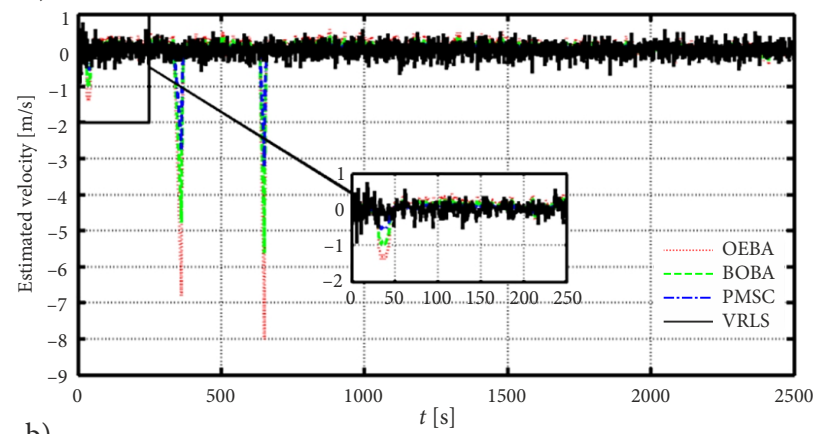

b)

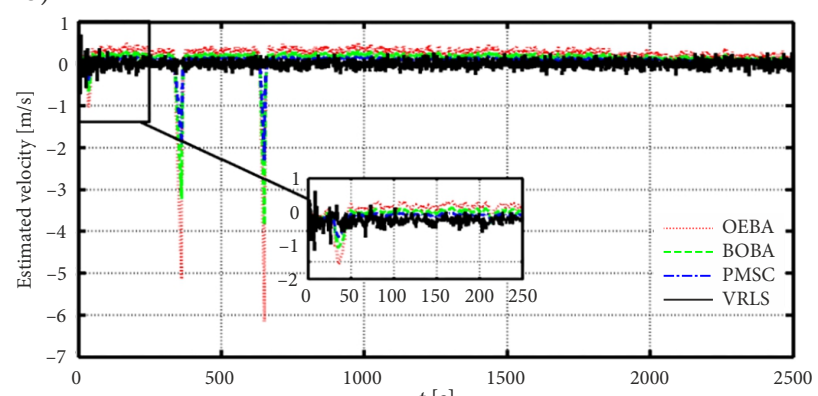

c)

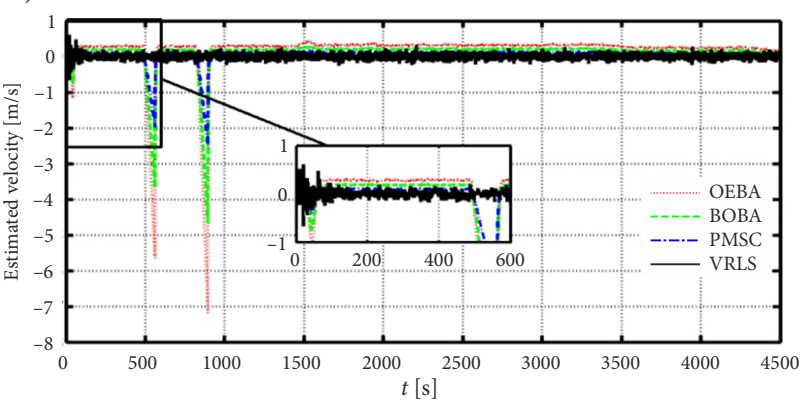

Figure 6. Estimations of velocity error for journeys with profiles: a - Journey-A with Profile-1; b - Journey-A with Profile-2; c - Journey-B with Profile-5

ing the velocity error with various accelerometer bias and noise.

Thus, it can be concluded from the analysis that for the assumed kinematic profile for the Journey-A and Journey-B, with various values of accelerometer bias and noise the velocity error estimated by the VRLS based adaptive multisensor data fusion technique comes out to be the least.

\subsubsection{Estimation of position error}

The performance of the proposed VRLS based adaptive multisensor data fusion technique for the estimation of position error is shown in Figure 7. The results shown in Figure 7 are only for the representative cases. So, the comparative performance of the fusion-filtering techniques for the estimation of position error for the assumed kinematic profile are tabulated in Table 4.

The techniques as discussed in researches by Acharya et al. (2011) and Ernest et al. (2004) used for the purpose of estimation of position and velocity errors have some limitations. The accuracy of both the techniques in the work reported by Ernest et al. (2004), i.e. the OEBA and 
Table 3. Comparative performance of the fusion-filtering techniques for velocity error estimation

\begin{tabular}{|c|c|c|}
\hline Journey name & Technique & $\begin{array}{c}\text { Absolute peak } \\
\text { velocity error }[\mathrm{m} / \mathrm{s}]\end{array}$ \\
\hline \multirow{4}{*}{$\begin{array}{l}\text { Journey-A } \\
\text { with accelerometer } \\
\text { bias }=0.20 \\
\text { accelerometer } \\
\text { noise }=0.05\end{array}$} & OEBA & 8.023 \\
\hline & BOBA & 5.616 \\
\hline & PMSC & 3.209 \\
\hline & VRLS (proposed) & 0.6918 \\
\hline \multirow{4}{*}{$\begin{array}{l}\text { Journey-A } \\
\text { with accelerometer } \\
\text { bias = } 0.26 ; \\
\text { accelerometer } \\
\text { noise }=0.02\end{array}$} & OEBA & 6.152 \\
\hline & BOBA & 3.845 \\
\hline & PMSC & 2.307 \\
\hline & VRLS (proposed) & 0.5645 \\
\hline \multirow{4}{*}{$\begin{array}{l}\text { Journey-A } \\
\text { with accelerometer } \\
\text { bias = } 0.30 ; \\
\text { accelerometer } \\
\text { noise }=0.05\end{array}$} & OEBA & 7.482 \\
\hline & BOBA & 5.985 \\
\hline & PMSC & 2.993 \\
\hline & VRLS (proposed) & 0.5394 \\
\hline \multirow{4}{*}{$\begin{array}{l}\text { Journey-B } \\
\text { with accelerometer } \\
\text { bias = } 0.10 ; \\
\text { accelerometer } \\
\text { noise }=0.02\end{array}$} & OEBA & 8.618 \\
\hline & BOBA & 5.171 \\
\hline & PMSC & 3.016 \\
\hline & VRLS (proposed) & 0.5887 \\
\hline \multirow{4}{*}{$\begin{array}{l}\text { Journey-B } \\
\text { with accelerometer } \\
\text { bias }=0.20 ; \\
\text { accelerometer } \\
\text { noise }=0.01\end{array}$} & OEBA & 7.188 \\
\hline & BOBA & 4.672 \\
\hline & PMSC & 2.516 \\
\hline & VRLS (proposed) & 0.4681 \\
\hline \multirow{4}{*}{$\begin{array}{l}\text { Journey-B } \\
\text { with accelerometer } \\
\text { bias }=0.25 \\
\text { accelerometer } \\
\text { noise }=0.01\end{array}$} & OEBA & 8.244 \\
\hline & BOBA & 6.229 \\
\hline & PMSC & 2.290 \\
\hline & VRLS (proposed) & 0.5294 \\
\hline
\end{tabular}

a)

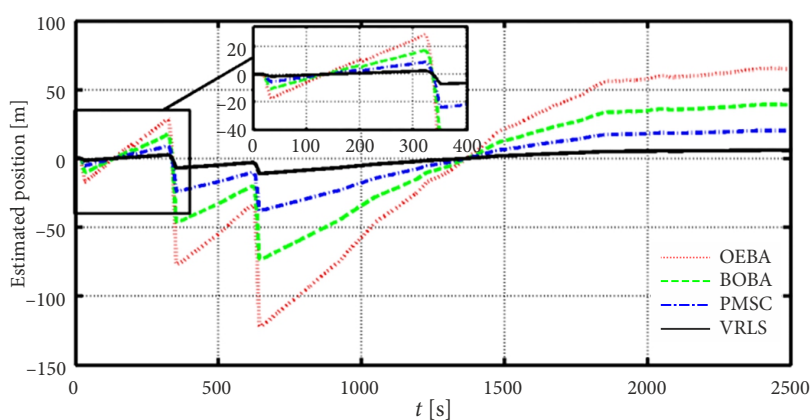

b)

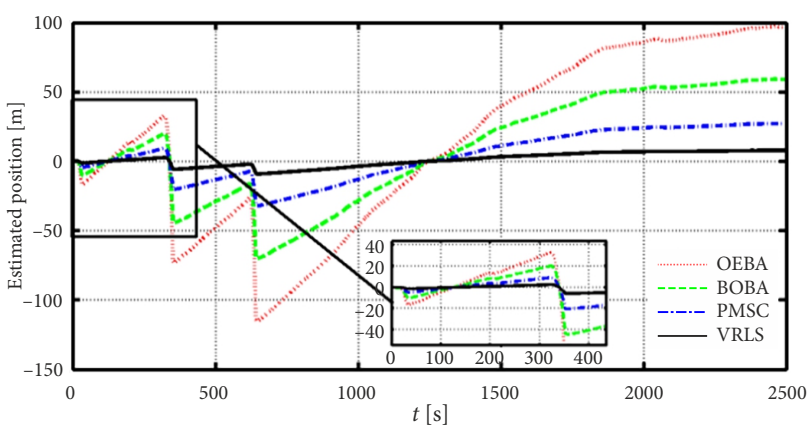

Table 4. Comparative performance of fusion-filtering techniques for position error estimation

\begin{tabular}{|c|c|c|c|}
\hline Journey name & Technique & $\begin{array}{l}\text { Absolute peak } \\
\text { position } \\
\text { error }[\mathrm{m}]\end{array}$ & $\begin{array}{c}\text { Final } \\
\text { position } \\
\text { error }[\mathrm{m}]\end{array}$ \\
\hline \multirow{4}{*}{$\begin{array}{l}\text { Journey-A } \\
\text { with accelerometer } \\
\text { bias }=0.20 \\
\text { accelerometer } \\
\text { noise }=0.05\end{array}$} & OEBA & 122.1 & -64.65 \\
\hline & BOBA & 73.13 & -38.88 \\
\hline & PMSC & 37.71 & -20.05 \\
\hline & $\begin{array}{l}\text { VRLS } \\
\text { (proposed) }\end{array}$ & 10.97 & -5.832 \\
\hline \multirow{4}{*}{$\begin{array}{l}\text { Journey-A } \\
\text { with accelerometer } \\
\text { bias }=0.26 \\
\text { accelerometer } \\
\text { noise }=0.02\end{array}$} & OEBA & 114.9 & -96.47 \\
\hline & BOBA & 69.72 & -58.85 \\
\hline & PMSC & 32.00 & -27.01 \\
\hline & \begin{tabular}{|l|} 
VRLS \\
(proposed)
\end{tabular} & 9.196 & -7.72 \\
\hline \multirow{4}{*}{$\begin{array}{l}\text { Journey-A } \\
\text { with accelerometer } \\
\text { bias }=0.30 \\
\text { accelerometer } \\
\text { noise }=0.05\end{array}$} & OEBA & 145.0 & -80.32 \\
\hline & BOBA & 84.56 & -46.85 \\
\hline & PMSC & 48.32 & -26.74 \\
\hline & \begin{tabular}{|l|} 
VRLS \\
(proposed)
\end{tabular} & 12.07 & -6.692 \\
\hline \multirow{4}{*}{$\begin{array}{l}\text { Journey-B } \\
\text { with accelerometer } \\
\text { bias }=0.10 \\
\text { accelerometer } \\
\text { noise }=0.02\end{array}$} & OEBA & 194.2 & -111.0 \\
\hline & BOBA & 121.4 & -69.56 \\
\hline & PMSC & 48.57 & -27.82 \\
\hline & \begin{tabular}{|l}
$\begin{array}{l}\text { VRLS } \\
\text { (proposed) }\end{array}$ \\
\end{tabular} & 19.52 & -11.10 \\
\hline \multirow{4}{*}{$\begin{array}{l}\text { Journey-B } \\
\text { with accelerometer } \\
\text { bias }=0.20 \\
\text { accelerometer } \\
\text { noise }=0.01\end{array}$} & OEBA & 211.5 & -163.3 \\
\hline & BOBA & 141.0 & -108.8 \\
\hline & PMSC & 56.21 & -43.55 \\
\hline & \begin{tabular}{|l}
$\begin{array}{l}\text { VRLS } \\
\text { (proposed) }\end{array}$ \\
\end{tabular} & 14.12 & -10.90 \\
\hline \multirow{4}{*}{$\begin{array}{l}\text { Journey-B } \\
\text { with accelerometer } \\
\text { bias }=0.25 \\
\text { accelerometer } \\
\text { noise }=0.01\end{array}$} & OEBA & 256.9 & -74.10 \\
\hline & BOBA & 154.0 & -44.47 \\
\hline & PMSC & 82.19 & -23.70 \\
\hline & \begin{tabular}{|l}
$\begin{array}{l}\text { VRLS } \\
\text { (proposed) }\end{array}$ \\
\end{tabular} & 30.83 & -8.893 \\
\hline
\end{tabular}

c)

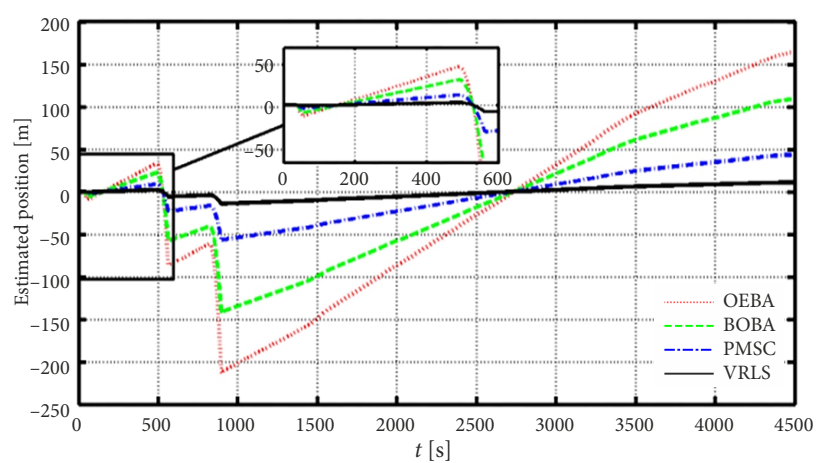

Figure 7. Estimations of position error for journeys with Profiles: a - Journey-A with Profile-1;

b - Journey-A with Profile-2; c - Journey-B with Profile-5 
BOBA mainly depends on choosing the appropriate value of filter parameters. In case of choosing an inappropriate value of the filter, parameters may result in an error in the estimation of position, which may grow with time.

In the work reported by Acharya et al. (2011), the limiting factor in case of the PMSC technique is noise. The navigation system while using in railway environments (due to slip and sliding of wheels) needs more accuracy and the noise may again give rise to an erroneous result in the estimation of position error. It thus becomes clear from Table 4, that the position error estimated by the proposed VRLS based adaptive multisensor data fusion technique outperforms for the chosen kinematic profiles for Journey-A and Journey- $B$, when compared with other works available in the literature.

\section{Conclusions}

In this work, a VRLS based adaptive multisensor data fusion technique is proposed for the TCAS used in Indian railways for the precise localization of trains during GPS outages.

For the TCAS in Indian railways, which works as an autonomous system the velocity and positional accuracy are the two major requirements. It is clear from the present analysis that the developed scheme is an effective one that can work with the TCAS during the GPS outages for the purpose of accurate train localization.

Future research work on this topic may concentrate on the real-time implementation and testing of the TCAS against various GPS receiver errors to further enhance the accuracy and robustness of the proposed technique.

\section{References}

Acharya, A.; Sadhu, S.; Ghoshal, T. K. 2011. Train localization and parting detection using data fusion, Transportation Research Part C 19(1): 75-84.

https://doi.org/10.1016/j.trc.2010.03.010

Arunachalam, S. S.; Guruprasad, M.; Koti, B. 2013. Performance of radar assisted satellite based automated train transit system, 2013 International Conference on Communication and Signal Processing, 3-5 April 2013, Melmaruvathur, India, 758-762. https://doi.org/10.1109/iccsp.2013.6577158

Bachmann, C.; Abdulhai, B.; Roorda, M. J.; Moshiri, B. 2012. Multisensor data integration and fusion in traffic operations and management, Transportation Research Record: Journal of the Transportation Research Board 2308: 27-36.

https://doi.org/10.3141/2308-04

Bajaj, R.; Ranaweera, S. L.; Agrawal, D. P. 2002. GPS: locationtracking technology, Computer 35(4): 92-94.

https://doi.org/10.1109/MC.2002.993780

Bar-Shalom, Y.; Li, X.-R. 1993. Estimation and Tracking: Principles, Techniques, and Software, Artech House. 511 p.

Chang, C. S.; Tsang, K. F. 2008. A cost effective approach on railway vehicle identification and positioning using RFID technology, IET 2nd International Conference on Wireless, Mobile and Multimedia Networks (ICWMMN 2008), 12-15 October 2008, Beijing, China, 490-493.

https://doi.org/10.1049/cp:20081043
Chen, Y.; Han, C.-Z. 2005. Maneuvering vehicle tracking based on multi-sensor fusion, Acta Automatica Sinica 31(4): 625-630.

Cooper, S.; Durrant-Whyte, H. 1994. A Kalman filter model for GPS navigation of land vehicles, in Proceedings of IEEE/RSJ International Conference on Intelligent Robots and Systems (IROS'94), 12-16 September 1994, Munich, Germany, 1: 157-163. https://doi.org/10.1109/IROS.1994.407396

Diniz, P. S. R. 2013. Adaptive Filtering: Algorithms and Practical Implementation. Springer. $652 \mathrm{p}$.

https://doi.org/10.1007/978-1-4614-4106-9

El Faouzi, N.-E.; Leung, H.; Kurian, A. 2011. Data fusion in intelligent transportation systems: progress and challenges - a survey, Information Fusion 12(1): 4-10. https://doi.org/10.1016/j.inffus.2010.06.001

Ernest, P.; Mazl, R.; Preucil, L. 2004. Train locator using inertial sensors and odometer, IEEE Intelligent Vehicles Symposium 2004, 14-17 June 2004, Parma, Italy, 860-865.

https://doi.org/10.1109/IVS.2004.1336497

Geistler, A.; Bohringer, F. 2004. Robust velocity measurement for railway applications by fusing eddy current sensor signals, in IEEE Intelligent Vehicles Symposium 2004, 14-17 June 2004, Parma, Italy, 664-669.

https://doi.org/10.1109/IVS.2004.1336463

Government of India. 2012. Handbook on Micro Controller Based Governor Fitted on Diesel Locomotives. CAMTECH/2012/M/ MCBG/1.0. Centre for Advanced Maintenance Technology (CAMTECH), Ministry of Railways, Government of India. $89 \mathrm{p}$.

Government of India. 2015. Indian Railways: Lifeline of the Nation. A White Paper. Government of India. Ministry of Railways, New Delhi, India. 66 p. Available from Internet: http://www. indianrailways.gov.in/railwayboard/uploads/directorate/finance_budget/Budget_2015-16/White_Paper-_English.pdf

Gregor, R.; Lutzeler, M.; Pellkofer, M.; Siedersberger, K.-H.; Dickmanns, E. D. 2002. EMS-Vision: a perceptual system for autonomous vehicles, IEEE Transactions on Intelligent Transportation Systems 3(1): 48-59.

https://doi.org/10.1109/6979.994795

Groves, P. D. 2013. Principles of GNSS, Inertial, and Multisensor Integrated Navigation Systems. Artech House. 776 p.

Hartwig, K.; Grimm, M.; Meyer zu Hörste, M.; Lemmer, K. 2006. Requirements for safety relevant positioning applications in rail traffic - a demonstrator for a train borne navigation platform called "DemoOrt", in WCRR - 7th World Congress on Railway Research, 4-8 June 2006, Montreal, Canada, 1-11.

Kalman, R. E. 1960. A new approach to linear filtering and prediction problems, Journal of Basic Engineering 82(1): 35-45. https://doi.org/10.1115/1.3662552

Kaplan, E. D. 1996. Understanding GPS: Principles and Applications. Artech House.

Khaleghi, B.; Khamis, A.; Karray, F. O.; Razavi, S. N. 2013. Multisensor data fusion: a review of the state-of-the-art, Information Fusion 14(1): 28-44.

https://doi.org/10.1016/j.inffus.2011.08.001

Kim, S.-B.; Bazin, J.-C.; Lee, H.-K.; Choi, K.-H.; Park S.-Y. 2011. Ground vehicle navigation in harsh urban conditions by integrating inertial navigation system, global positioning system, odometer and vision data, IET Radar, Sonar \& Navigation 5(8): 814-823. https://doi.org/10.1049/iet-rsn.2011.0100

Malakar, B.; Roy, B. K. 2014. Survey of RFID applications in railway industry, in 2014 First International Conference on Automation, Control, Energy and Systems (ACES), 1-2 February 2014, Hooghy, India, 1-6.

https://doi.org/10.1109/ACES.2014.6807999 
Mangal, M. 2013. Specific requirements and options for CCS of Indian railways: train collision avoidance system

(TCAS), in UIC Global Signalling \& Telecom Conference, 2123 March 2013, Delhi, India.

Mansukhani, L. 2014. Train collision avoidance system (TCAS), IRSTE Journal 1: 11-18.

Mirabadi, A.; Sandidzadeh, M. A.; Hosseingholian, M.; Schmid, F. 2002. Fault tolerant train navigation system via integration of GPS, balises, tachomters and Doppler radar, in 2002 AREMA Conference, 23-25 September 2002, Washington, DC, US, $1-27$.

Mirabadi, A.; Schmid, F.; Mort, N. 2003. Multisensor integration methods in the development of a fault-tolerant train navigation system, The Journal of Navigation 56(3): 385-398. https://doi.org/10.1017/S0373463303002364

Mitchell, H. B. 2007. Multi-Sensor Data Fusion: an Introduction. Springer. 282 p. https://doi.org/10.1007/978-3-540-71559-7

Noureldin, A.; Karamat, T. B.; Georgy, J. 2013. Fundamentals of Inertial Navigation, Satellite-Based Positioning and their Integration. Springer. $314 \mathrm{p}$. https://doi.org/10.1007/978-3-642-30466-8

Prakash, G.; Meena, F. S.; Prakash, A. 2008. Problem in tunneling in railway projects with special reference to North Eastern region of India, in Underground Facilities for Better environment \& Safety: ITA-AITES World Tunnel Congress \& 34th General Assembly, 19-25 September 2008, Agra, India.

Qin, H.; Cong, L.; Sun, X. 2012. Accuracy improvement of GPS/ MEMS-INS integrated navigation system during GPS signal outage for land vehicle navigation, Journal of Systems Engineering and Electronics 23(2): 256-264.

https://doi.org/10.1109/JSEE.2012.00033

Ribeiro, M. I. 2004. Kalman and Extended Kalman Filters: Concept, Derivation and Properties. Institute for Systems and Robotics, University of Lisbon, Portugal. $45 \mathrm{p}$.

Santos, A. J. D.; Soares, A. R.; De Almeida Redondo, F. M.; Carvalho, N. B. 2005. Tracking trains via radio frequency systems, IEEE Transactions on Intelligent Transportation Systems 6(2): 244-258. https://doi.org/10.1109/TITS.2005.848369

Walts, E. L. 1988. Data fusion for C3I: a tutorial, in The C3I Handbook - Command Control Communications Intelligence, 217-226.

Wang, W. J.; Wang, H. Y.; Guo, J.; Liu, Q. Y.; Zhu, M. H.; Jin, X. S. 2014. Experimental investigation of adhesion coefficient of wheel/rail under the track ramp conditions, Proceedings of the Institution of Mechanical Engineers, Part J: Journal of Engineering Tribology 228(7): 808-815. https://doi.org/10.1177/1350650114526386

Zhang, X.; Lakafosis, V.; Traille A.; Tentzeris, M. M. 2010. Performance analysis of "fast-moving" RFID tags in state-of-the-art high-speed railway systems, 2010 IEEE International Conference on RFID-Technology and Application, 17-19 June 2010, Guangzhou, China, 281-285.

https://doi.org/10.1109/RFID-TA.2010.5529918

Zhao, Y. 2011. GPS/IMU Integrated System for Land Vehicle Navigation based on MEMS. Licentiate Thesis in Geodesy. KTH Royal Institute of Technology, Stockholm, Sweden. $92 \mathrm{p}$. Available from Internet: http://kth.diva-portal.org/smash/record.jsf?pid=diva2\%3A446078\&dswid=-246 